\section{Individuelle Labortests unter Isotretinoin}

\begin{abstract}
Aknepatienten unter oraler IsotretinoinTherapie in Standarddosierung können sich künftig möglicherweise einige Blutentnahmen sparen. Bei der Therapie drohen schwere Nebenwirkungen. US-Ärzte sehen bei vielen Patienten mit Standarddosierung dennoch keinen Grund für monatliche Kontrollen. In einer Metaanalyse konnten sie zwar signifikante Veränderungen bei Leukozyten, Leber- und Lipidwerten unter Isotretinoin bestätigen. Diese signalisierten aber kein generell hohes Risiko, so die Autoren. Die Tests sollten in Abhängigkeit vom Ausgangswert und unter Berücksichtigung von Komorbiditäten und weiteren leberbelastenden Medikamente individuell festgelegt werden.
\end{abstract}

Lee Y Het al. JAMA Dermatology 2015 (online first)

\section{Mikrozephalie durch Zika-Viren?}

Brasilien hat den Gesundheitsnotstand ausgerufen: Es gibt eine Häufung von Schädelfehlbildungen bei Neugeborenen. Ursache ist womöglich eine Zika-Virusinfektion, die über Mückenstiche auf Schwangere übertragen wird. 399 Fälle in sieben Bundesstaaten wurden registriert, ein deutlicher Anstieg gegenüber Vorjahren. Reisende sollten sich sorgfältig vor den Überträgermücken schützen, empfiehlt das CRM Centrum für Reisemedizin - und zwar in allen tropischen und subtropischen Regionen.

www.aerztezeitung.de

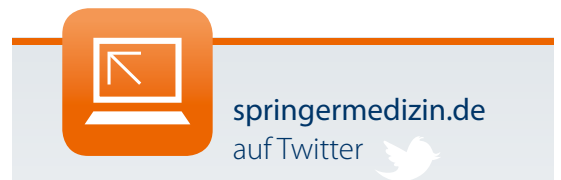

Auch im Advent heißt es

"Lauschen" Sie unserem

Zwitschern und werden Sie zum

"Follower"

- twitter.com/springermedizin

Aufs Alter kommt's an

\title{
Schilddrüsenknoten: Wie oft entarten sie?
}

\section{Die Zahl der Schilddrüsenknoten nimmt zwar im Alter zu, aber deren Malignitätsrisiko verringert sich zu- gleich. Das lässt sich aus einer pros- pektiven US-Studie über einen Zeitraum von fast zwei Dekaden ablesen.}

An der Studie nahmen fast 6400 euthyreote Patienten im Alter zwischen 20 und 95 Jahren teil, die bei Verdacht auf Schilddrüsenknoten sonografisch untersucht wurden. Dokumentiert wurden alle Knoten mit einem Mindestdurchmesser von einem Zentimeter. Je älter die Patienten mit Schilddrüsenknoten waren, umso mehr Knoten wurden in dem Organ entdeckt. So hatten die Patienten der jüngsten Altersgruppe (20-29 Jahre) durchschnitt- lich 1,55 Knoten $\geq 1 \mathrm{~cm}$, in der höchsten Altersgruppe ( $\geq 70$ Jahre) dagegen durchschnittlich 2,21 Knoten.

\section{Krebsprävalenz sank auf 12,6\%}

Im Verlauf der 16 Jahre dauernden Studie erkrankten 1018 Patienten (15,9\%) an einem Schilddrüsenkarzinom. Die Krebsprävalenz sank von $22,9 \%$ in der jüngsten Altersgruppe auf 12,6\% in der ältesten Teilnehmergruppe. Nach Angaben der Endokrinologen und Pathologen sank damit das relative Risiko für maligne Knoten zwischen 20 und 60 Jahren um $2,2 \%$ jährlich. Danach sei das Risiko gleichgeblieben.

(ple)

Kwong N et al. JCEM 2015; online 14. Oktober.

doi: 10.1210/jc.2015-3100X

\section{Vor allem Frauen sind betroffen}

\section{Hypertonie ist mäßig mit Hörverlust assoziiert}

Bei Frauen ist Hypertonie zwar mit einem leicht erhöhten Risiko für Hörverlust verbunden. Allerdings ohne Assoziation zwischen den Höreinbußen und der Therapie mit Thiaziddiuretika oder Furosemid.

HNO-Ärzte aus Boston untersuchten den Zusammenhang zwischen der Behandlung mit Diuretika und Hörverlust anhand der der Daten aus der Nurses Health Study I von über 54.000 Frauen. Fast jede zweite Teilnehmerin (47\%) gab bei einer Befragung 2012 an, Probleme mit dem Hören zu haben. Fast jede Dritte (31\%) hatte im Studienzeitraum Bluthochdruck. 18\% dieser Frauen erhielten ausschließlich Thiaziddiuretika, 3\% nur Furosemid und 0,1\% beide Diuretikaarten. Die kumulative Inzidenz des Hörverlusts lag bei 35\%. Dabei stellten die Studienautoren eine unabhängige, aber nur mäßige Assoziation zwischen Hypertonie und Hörverlust-Risiko fest. Bezugspunkt waren Frauen ohne Hypertonie.

Ebenfalls gab es, bei Berücksichtigung aller Frauen, eine unabhängige Assoziation zwischen Thiaziddiuretikabehandlung und Hörverlust (RR: 1,08), nicht jedoch bei der Therapie mit Furosemid (RR: 1,01). Beschränkt man die Analyse auf Teilnehmerinnen mit Hypertonie, gab es keinerlei Assoziation, wie die Ärzte berichten. Das war auch der Fall, wenn die Dauer der Therapie mit Antihypertensiva und Diuretika sowie Kofaktoren wie BMI, Hüftumfang und Tinnitus bei der Analyse berücksichtigt wurden. (p/e)

Lin BM et al. Hypertension. Am J Med 2015, online 30. November 